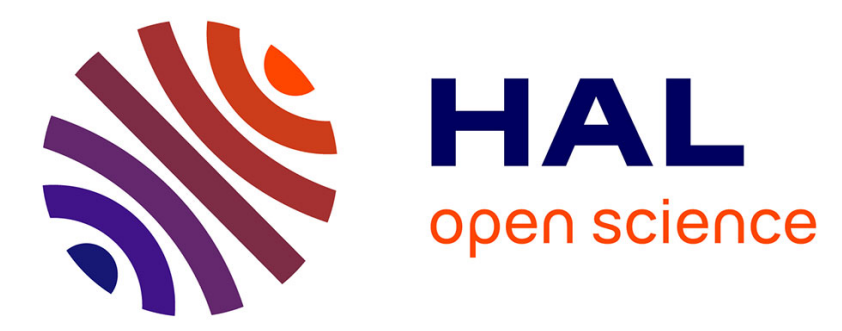

\title{
Antigenic features of protein carriers commonly used in immunisation trials
}

Pieranna Chiarella, Bärbel Edelmann, Vito M. Fazio, Alan M. Sawyer, Ario

Marco

\section{> To cite this version:}

Pieranna Chiarella, Bärbel Edelmann, Vito M. Fazio, Alan M. Sawyer, Ario Marco. Antigenic features of protein carriers commonly used in immunisation trials. Biotechnology Letters, 2010, 32 (9), pp.12151221. 10.1007/s10529-010-0283-z . hal-00589680

\section{HAL Id: hal-00589680 \\ https://hal.science/hal-00589680}

Submitted on 30 Apr 2011

HAL is a multi-disciplinary open access archive for the deposit and dissemination of scientific research documents, whether they are published or not. The documents may come from teaching and research institutions in France or abroad, or from public or private research centers.
L'archive ouverte pluridisciplinaire HAL, est destinée au dépôt et à la diffusion de documents scientifiques de niveau recherche, publiés ou non, émanant des établissements d'enseignement et de recherche français ou étrangers, des laboratoires publics ou privés. 


\section{ANIMAL CELL TECHNOLOGY}

Antigenic features of protein carriers commonly used in immunisation trials

Pieranna Chiarella ${ }^{1}$, Bärbel Edelmann ${ }^{2}$, Vito M. Fazio ${ }^{1}$, Alan M. Sawyer ${ }^{3}$ and Ario de $\operatorname{Marco}^{4^{*}}$

${ }^{1}$ Laboratory of Molecular Medicine and Biotechnology. CIR, Unive rsity of Rome Campus Bio-medico. Via Alvaro del Portillo 21 - 00128 Rome, Italy

${ }^{2}$ Institut für Immunologie, Universität Kiel, Michaelisstr. 5 - 24105 Kiel, Germany ${ }^{3}$ Monoclonal Antibody Core Facility. Mouse Biology Programme - EMBL, Via Ramarini 32 - 00015 Monterotondo Scalo, Italy ${ }^{4}$ Cogentech, IFOM-IEO Campus, Via Adamello 16 - 20139 Milan, Italy

${ }^{*}$ Corresponding author: Cogentech, via Adamello, 16 - 20139, Milano, Italy. Tel. 0039.02.574303704, Fax.0039.02.574303231, ario.demarco@ifom-ieo-campus.it 


\section{Purpose of work}

Short-peptide immunisation remains a trial-and-error procedure; however, single components of the protocol, like the rational choice of carriers and adjuvants that can influence the animal immune response, can be optimized.

\section{Abstract}

An aluminium hydroxide adjuvant induced a more elevated and rapid immune responses against short peptides conjugated to the Keyhole Lympet Hemocyanin carrier than immuneasy adjuvant. Furthermore, since carrier proteins may compete with the fused or chemically linked polypeptides in eliciting antigen-specific immune response, we classified the immunogenicity of the most common carrier proteins used in molecular biology for antigen expression and mouse immunisation. The disulfide isomerase protein A gave a carrier with the lowest immunogenicity whilst disulfide isomerase protein $\mathrm{C}$ gave the highest immunogenicity and therefore should be avoided as a fusion partner. Using this protein as a model, we identified and located the immunodominant epitopes along its sequence. These results now enable the combination of carrier and immunisation conditions to be optimized.

Key-words: affinity tags, disulfide isomerase protein C, epitope mapping, hybridoma technology, monoclonal antibodies, peptide immunisation

\section{Introduction}


Immunisation of animals with appropriate antigen formulations is fundamental to achieve suitable humoural immune responses. The choice of appropriate adjuvants is crucial for optimising antigen delivery and stimulation of the immune system (Mutwiri et al. 2007; Chiarella et al. 2007; Wilson-Welder et al. 2009), especially in the case of short peptides where the immunogenicity is often deceptively low. To improve their immunogenicity, peptides must be chemically conjugated or engineered by means of molecular biological techniques to carrier proteins to stimulate the B lymphocyte-mediated response sufficiently, and increase the immunogens in vivo half-life (Kirkley et al. 2001).

Peptides are either coupled to carriers, such as bovine serum albumin or ovalbumin, or fused to tag proteins, such as glutathione-S-transferase (GST) and maltose binding protein (MBP), which may also facilitate the affinity purification of the fusion constructs (De Marco et al. 2004). Although immunisation with tagged-antigens stimulates the overall immune response of the host (Herzenberg et al. 1980; Di John et al. 1989), large tags may show immunodominance over the target antigen and strongly inhibit the immune response specific for the target antigen (Schutze et al. 1989; Sad et al. 1991).

In this paper we report some results of practical benefit for planning immunisation trials aimed at obtaining valuable monoclonal antibodies by means of hybridoma cell technology.

\section{Material and Methods}

Antigens, reagents and robotics 
Disulfide isomerase protein $\mathrm{A}(\mathrm{DsbA})$, disulfide isomerase protein $\mathrm{C}$ (DsbC), maltose binding protein (MBP), glutathione-S-transferase (GST), transcription termination factor (NusA), thioredoxin (Trx), and SNAP were produced by means of pETM vectors (Dümmler et al. 2005; Bossi et al. 2010) and used for the tag immunogenicity assay. FLAG (DYKDDDDK), HA (YPYDVPDYA), MYC (EQKLISEEDL), and VSV-G (YTDIEMNRLGK) peptides were from Sigma and coupled to keyhole limpet hemocyanin $(\mathrm{KLH})$ through $C$-terminal cysteine by using Imject maleimide-activated mcKLH (Pierce). The tissue culture robot was supplied by Tecan A.G. and the MicroGrid II 600 arrayer from Apogent Discoveries.

Immunisation protocols

Six-week old female Balb/C mice were immunised by intraperitoneal and subcutaneous injections with $10 \mu \mathrm{g}$ antigen at 14 day intervals. Immuneasy adjuvant (Qiagen) and aluminium hydroxide adjuvant (alum) (Pierce), plus $10 \mathrm{nmol}$ CpG-DNA per mouse (Krieg 1990) were used for antigen preparation. Blood was harvested 1012 days after boosting and centrifuged for 5 min at 4500xg for antiserum collection. Glycerol was added to $80 \%(\mathrm{v} / \mathrm{v})$ and stored at $-20^{\circ} \mathrm{C}$. The antibody titre was evaluated by ELISA. Four days prior to fusion, each mouse was immunised with the same antigen formulation and sacrificed on day 5 by cervical dislocation. For antigen pre-treatment, half of protein was held at $98^{\circ} \mathrm{C}$ for $5 \mathrm{~min}$, to make more cryptic epitopes available and then mixed with the native antigen prior to immunisation.

Hybridoma generation and ELISA analysis

Mouse splenocytes were obtained by mechanical disruption of the spleen and filtered into a $50 \mathrm{ml}$ tube through a $70 \mu \mathrm{m}$ nylon cell strainer before being centrifuged 
at $170 \times$. Red blood cells were eliminated by incubating the cell suspension with Red Cell Lysis Buffer (Sigma) for a few minutes at room temperature. The lysis was stopped by adding HM20 (DMEM, 20\% v/v foetal bovine serum (Hyclone Defined), 2 mM L-glutamine, $50 \mu \mathrm{M}$ gentamicin. Splenocytes were then centrifuged at $170 \mathrm{xg}$ and resuspended in DMEM, counted, and mixed with SP2 myeloma cells at 5:1 ratio. Polyethyleneglycol 1500 was added dropwise over $1 \mathrm{~min}$ at $37^{\circ} \mathrm{C}$ with agitation. Somatic cell fusion and antigen microarray assay were performed as previously described by De Masi et al. (2005).

ELISA was performed in 96-well plates (Maxisorp, Nunc) using $50 \mu \mathrm{L}$ hybridoma supernatant and immune serum at dilutions indicated in the figure legends. Secondary anti-mouse lgG alkaline phosphatase-conjugated antibodies (Jackson Immunoresearch) were diluted 1:5000 in PBS and p-nitrophenyl phosphate (Sigma) was used as a substrate. Three independent experiments were performed for each antigen/antibody pair.

\section{Epitope mapping}

DsbC fragments were produced by PCR and the resulting sequences were cloned in frame into the pETM33 vector to generate GST-fusion constructs (Dümmler et al. 2005), whilst proteins were chemically fragmented using NTCB (Fluka) according to Tang and Speicher (2004). Generated polypeptides were separated by electrophoresis using a 15\% acrylamide gel, blotted on nitrocellulose and hybridoma supernatants diluted 1:500 were used as a source of primary antibodies for protein specific recognition. Detection was performed using HRP-conjugated goat antimouse antibodies. 


\section{Results}

Optimisation of immunisation protocols for short tag peptides The peptides corresponding to the four tags HA, MYC, FLAG, VSVG were conjugated to KLH and either emulsified with Immuneasy and CpG-DNA or with alum adjuvant plus CpG-DNA for immunisation. The ELISA assays (Fig. 1) show that mice immunised with alum adjuvant were already responsive after the first boost and antibody levels in the serum peaked in the second bleed (black lines). In contrast, mice immunised with the same antigens emulsified in immuneasy adjuvant were not initially stimulated and a significant response was induced only by repeated injections of HA and MYC antigens, whereas FLAG and VSVG immunisation completely failed (dashed lines).

Tag-fusion protein immunogenicity study

The most common carriers used to stabilise fusion-protein constructs were injected into mice and their polyclonal sera were harvested and assayed by ELISA. As shown in Figure 2, the tag that induced the lowest antibody response ( $\lg M$ and $\lg G$ ) was DsbA, followed by GST, MBP, TRX, NusA and DsbC.

The monoclonal antibodies recovered from these experiments, all positive by immunoblot when challenged against the corresponding full-length antigen, were used to evaluate whether the higher immune response values correlated with the presence of particularly immunogenic epitopes on the protein carriers. First, these were chemically fragmented, immunoblotted and tested using the supernatant from fifteen independent hybridoma cell lines for each antigen. Selected data from the highly immunogenic DsbC are reported as an example. Most of the supernatants 
recognise variable protein fragment patterns (Fig. $3 \mathrm{~A}$ ), indicating that they are specific for independent epitopes. In an attempt aimed at further characterising the exact epitopes recognised by each cell line, the capacity of the hybridoma supernatants to detect specific antigen regions by immunoblot was compared as shown in Figure 3. In the case of DsbC, five regions were cloned as fusion constructs to GST and the resulting purified proteins used for immunoblotting. When clone 14 was used, a signal was detected only in three of the five samples (Fig. 3B). This information was used to infer the minimal common region among the positive samples corresponding to the sequence (aa 87-119) containing the epitope for that specific antibody (Fig. 3C). The same experimental approach was repeated for the other DsbC clones and these data are summarised in Table 1.

This indicates that DsbC immunogenicity results from the combination of several active epitopes rather than from a strong response induced by a unique epitope. A similar complexity was observed when the hybridoma against NusA and MBP were analysed using the same technique. In contrast, proteins such as DsbA and GST that raise low immunogenic response seem also to stimulate a reaction limited to a single epitope (data not shown).

DsbA carrier increases immunogenicity of the fused SNAP peptide The experiments reported above indicate that carrier proteins can induce significantly different immune-responses. Starting from this knowledge, we wished to understand whether this information could be of practical utility and, therefore, we prepared two constructs in which the SNAP-tag was fused to DsbA (low antigenicity) and $\mathrm{DsbC}$ (high antigenicity), respectively. The recombinant proteins were used for immunisation using the previously optimised protocol (Fig. 1) and the resulting anti- 
sera were examined for evaluating the immune-response. The DsbC-SNAP construct induced the highest response, but most of the immunoactivity was raised against the carrier, instead of the tag peptide (Fig. 4). In contrast, the specific antiSNAP response was more elevated in the mice immunised with DsbA-SNAP, despite the lower overall response (Fig. 4).

\section{Discussion}

Short peptide sequences, such as those corresponding to HA, MYC, FLAG, VSVG tags, are commonly used for affinity chromatography, western blotting, immunopurification or in vivo detection of the recombinant proteins to which they are fused. Identifying proteins with anti-tag antibodies may represent an effective alternative to generating specific antibodies to each single recombinant protein. Nevertheless, the immunogenicity of short peptides is low. The chances for success may be significantly improved if the optimal immunisation conditions are identified. We initially compared immunisation protocols using peptides chemically conjugated to $\mathrm{KLH}$ and procedures differing in the number of injections and antigen formulation. In our hands alum adjuvant was more effective for both stimulating the immune reaction and shortening the immunisation protocol.

There are however several technical and economic reasons for which researchers may prefer to clone polypeptides in frame with carrier proteins and to produce the fusion construct instead of preparing chemical conjugates. Most of the antigens provided by investigators with the aim of producing monoclonal antibodies are expressed in Escherichia coli and derive from fusion constructs in which the antigen is linked to the $\mathrm{N}$ - or $\mathrm{C}$-terminus of a carrier protein. Such fusion proteins are used to 
improve the solubility of the passenger polypeptides, to simplify the fusion construct purification and may contribute to enhance the overall immune response (Herzenberg et al. 1980; Di John et al. 1989). In our experience, immunisations with antigens fused to carrier proteins are reliable in terms of antibody production specific to the target antigen. However, their specific immunogenicity must be low to prevent immunodominance with respect to the target antigen (Schutze et al. 1989). Consequently, it is crucial to identify what, among the commonly used carrier proteins (Dümmler et al. 2005), should be avoided for immunisation applications due to their elevated immunogenicity and whether immunisation conditions can improve the immune response towards the tag without increasing the detrimental response against the carrier. Therefore, in a first experimental set we have systematically analysed the carrier's immunogenic features looking for acceptable levels of immunogenicity and showed that DsbA has the lowest immunogenicity among the carriers present in the panel. GST and MBP still possessed acceptable immunogenicity, whereas NusA, DsbC and TRX were the most immunogenic tags and should not be used for producing fusion proteins for immunisation to avoid that carrier immunodominance limits a suitable immune-response towards the target polypeptide, as in the case of the DsbC-SNAP construct. Finally, we could show that the combination of correct carrier choice (Fig. 2) and optimised immunisation procedures, such as those inferred by the results reported in Figure 1, enabled to identify a suitable protocol for obtaining specific anti-tag antibodies from animals immunised with DsbA-SNAP fusion proteins (Fig. 4).

Very often the immune response is focussed against a single immunodominant epitope. The epitope mapping data presented here indicated that, at least for some carriers such as DsbC and NusA, there are multiple regions capable of inducing an 
immune response. In contrast, proteins like DsbA and GST possess a single immunogenic region. This observation may be exploited for improving the carrier features by directed mutations of the crucial residues responsible for immunogenicity and, therefore, engineering the carriers to completely silence their residual immunogenicity.

\section{Acknowledgments}

The authors wish to thank N. Rosenthal and C. Boulin for having made accessible the EMBL Facilities and M. Leuener and C. Fasci for technical assistance. PC is supported by FIRB-Idee Progettuali RBIP0695BB_001.

\section{References}

Bossi S, Ferranti B, Martinelli C, Capasso P, de Marco A (2010) Antibody-mediated purification of co-expressed antigen-antibody complexes. Prot Express Purif doi:10.1016/j.pep.2010.01.003.

Chiarella P, Massi E, De Robertis M, Signori E, Fazio VM (2007) Adjuvant in vaccines and for immunisation: current trends. Exp Opin Biol Ther 7:15511562.

De Marco V, Stier G, Blandin S, de Marco A (2004) Comparison of NusA and GST as fusion partners for recombinant expression in E. coli. Biochem Biophys Res Commun 322:766-771.

De Masi F, Chiarella P, Wilhelm H, Massimi M, Bullard B, Ansorge W, Sawyer A (2005) High-throughput mouse monoclonal antibodies using antigen microarrays. Proteomics 5:4070-4081. 
Di John D, Torres JR, Murillo J, Herrington DA., et al. (1989) Effect of priming with carrier on response to conjugate vaccine. Lancet 2: 1415-1418.

Dümmler A, Lawrence AM, de Marco A (2005) Simplified screening for the detection of soluble fusion constructs expressed in E. coli using a modular set of vectors. Microbial Cell Factories 4:34.

Herzenberg LA, Tokuhisa T, Herzenberg LA (1980) Carrier-priming leads to haptenspecific suppression. Nature 285:664-667.

Kirkley JE, Goldstein AL, Naylor PH (2001) Effect of peptide-carrier coupling on peptide-specific immune response. Immunobiology 203:601-615.

Krieg AM (1990) Mechanisms and applications of immune stimulatory CpG oligodeoxynucleotides. Biochim Biophys Acta 1489, 107-116.

Mutwiri G, Gerdts V, Lopez M, Babiul LA (2007) Innate immunity and new adjuvants. Rev Sci Tech 26:147-156.

Sad S, Gupta HM, Talwar GP, Raghupathy R (1991) Carrier induced suppression of the antibody response to a "self" hapten. Immunology 74:223-227.

Schutze MP, Deriaud E, Przewlocki G, LeClerc C (1989) Carrier-induced epitopic suppression is initiated through clonal dominance. J Immunol 142:26352640.

Tang HY, Speicher DW (2004) Identification of alternative products and optimization of NTCB cyanylation and cleavage at cysteine residues. Anal Biochem 334:48-61.

Wilson-Welder JH, Torres MP, Kipper MJ, Mallapragada SK, Wannemuehler MJ, Narasimhan B (2009) Vaccine adjuvants: current challenge and future approaches. J P harm Sci 98:1278-1316. 
Table 1. Epitope mapping of anti-DsbC antibodies.

\begin{tabular}{cc}
\hline Epitope (initial and terminal aa) & Number of hybridoma cell lines \\
$1-20$ & 7 \\
$21-43$ & 1 \\
$44-86$ & 1 \\
$87-119$ & 1 \\
$120-152$ & 0 \\
$153-211$ & 5 \\
\hline
\end{tabular}

Immunoblotting was used to identify the minimal DsbC amino acid region specifically recognised by the supernatant recovered from fifteen independent hybridoma cell lines. 


\section{Figure Legends}

Figure 1. Adjuvant-dependent immune response of mice challenged with peptides fused to KLH.

Mice were immunised every two-weeks with KLH-peptides and CpG-DNA plus either immuneasy (dashed lines) or alum adjuvant (black lines). Blood samples were recovered 12-14 days after boosting and anti-sera were assayed using the uncoupled peptides diluted 1:500. PI: pre-immune serum.

Figure 2. Immunogenicity of carrier proteins.

Mice were immunised with $10 \mu \mathrm{g}$ of carrier protein in the presence of immuneasy adjuvant and CpG-DNA. A total number of four immunisations was performed and mice were bled 12-14 days after the second (1st bleed), the third ( $2^{\text {nd }}$ bleed), and the fourth (pre-fusion) immunisation. Sera were tested by ELISA using either anti-mouse $\lg M$ or anti-mouse $\lg G$ secondary antibodies and the colour reaction was measured as optical density at $405 \mathrm{~nm}$.

Figure 3. DsbC epitope mapping.

A) DsbC was chemically fragmented, blotted and tested using the supernatant recovered from independent hybridoma cell lines generated against this tag protein. Differential recognition patterns (A, B, C, D, E) were identified.

B) The anti-DsbC hybridoma clone 14 was used for staining blotted fusion constructs of GST plus DsbC specific regions. The letters indicate the following DsbC amino acid sequences: a (1-152); b (1-86); c (1-43); d (44152); e (21-119).

C) The epitope recognised by clone 14 was identified as the minimal common sequence among the western blot positive constructs. 
Figure 4. Immune response against carrier+SNAP tag constructs.

Diluted sera (1:200) recovered from mice immunised with DsbA-SNAP and DsbCSNAP fusion proteins were analysed by ELISA. The plates were coated with either the same fusion proteins used for immunisation or with the purified SNAP tag polypeptide. 
Figure 1

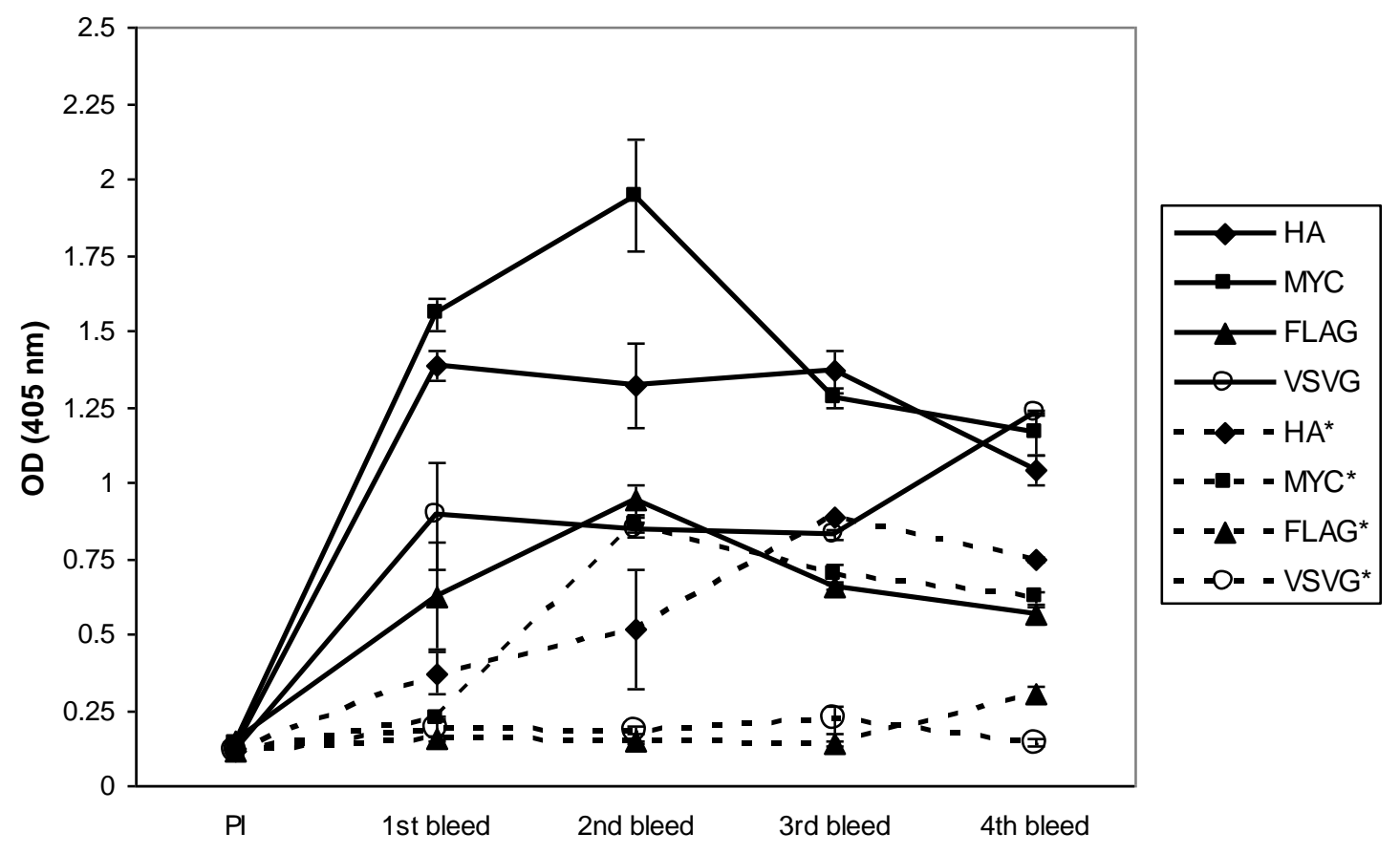


Figure 2
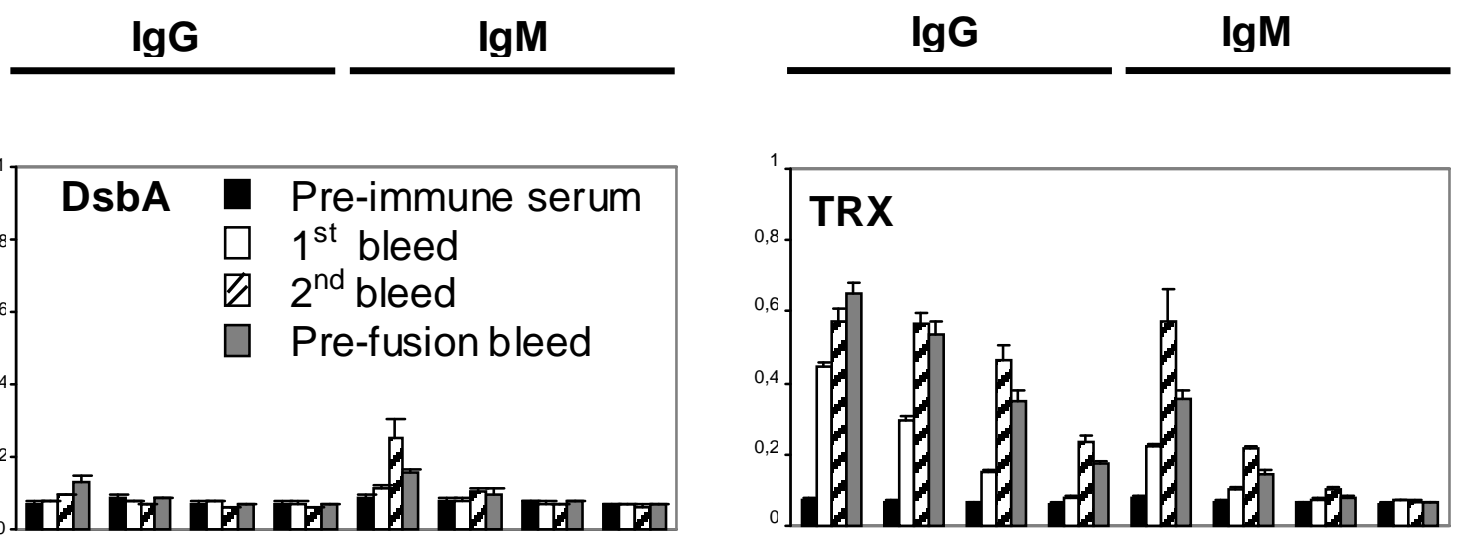

OD $405 \mathrm{~nm}$
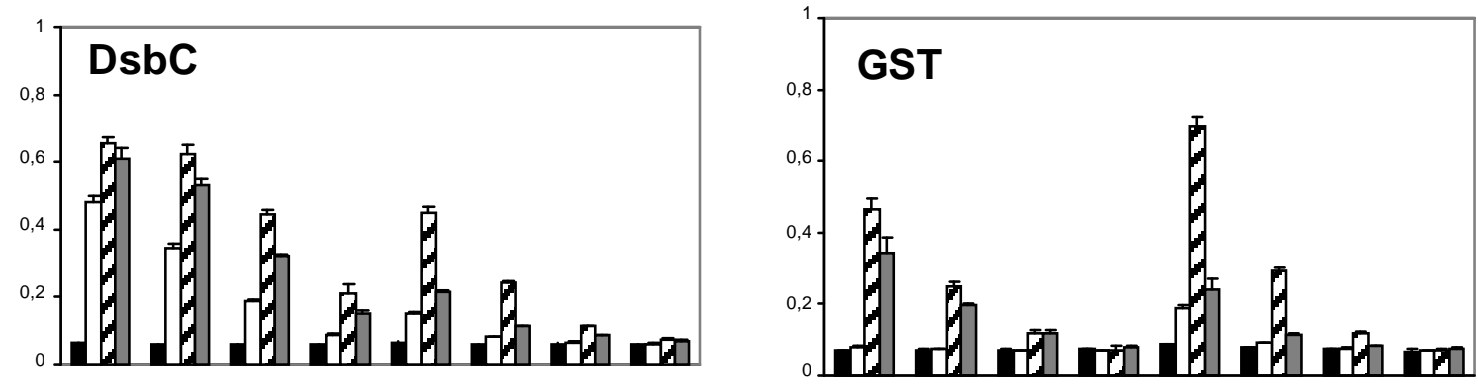

OD $405 \mathrm{~nm}$
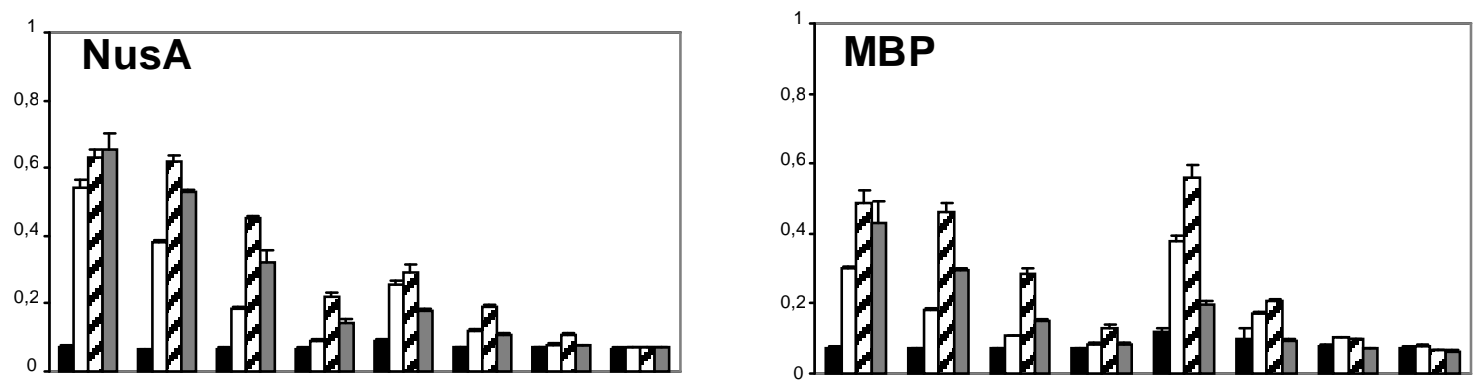
Figure 3

A) $\quad A \quad B \quad C \quad D \quad A \quad E$

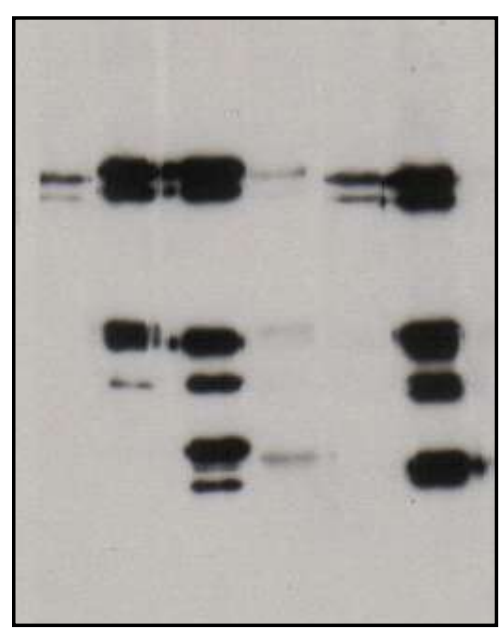

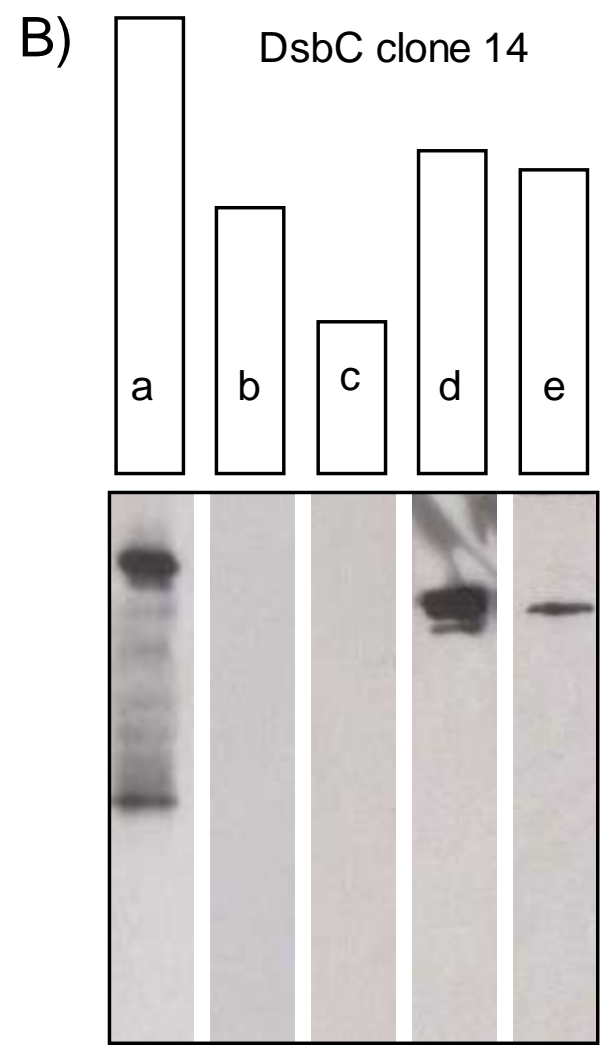


C)

\begin{tabular}{|c|c|c|}
\hline \multicolumn{3}{|c|}{$87-119$} \\
\hline $1-211$ & & \\
\hline $1-152$ & & \\
\hline $1-86$ & & \\
\hline $1-43$ & & \\
\hline 44 & - & 152 \\
\hline $21-119$ & & \\
\hline
\end{tabular}


Figure 4

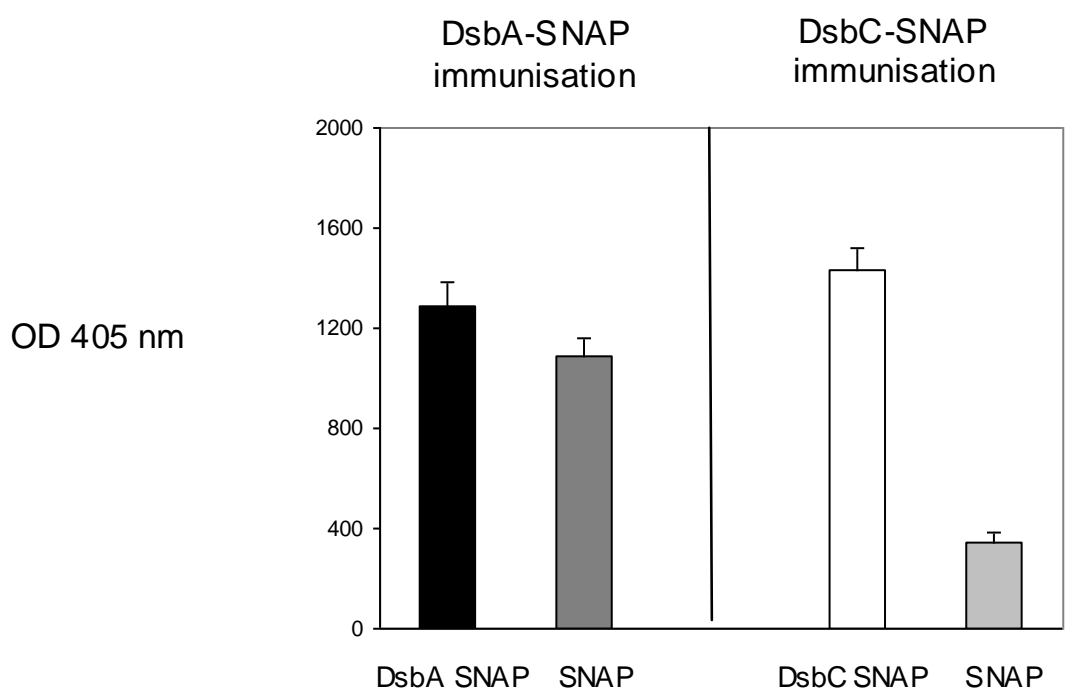

